\title{
IMMUNOLOGY
}

\section{Clonal deletion contributes to allograft tolerance}

A new assay to identify donorreactive $T$ cells and track them after organ transplantation has provided evidence for clonal deletion as a mechanism of allograft tolerance in recipients of combined kidney and bone marrow transplants (CKBMT). The assay, designed by Megan Sykes and colleagues, uses next-generation sequencing to define the 'fingerprint' of donor-reactive T cells.

Sykes and colleagues' previous research in a small cohort of tolerant CKBMT recipients had suggested that regulatory T cells might be involved in early tolerance, whereas clonal deletion of donor-reactive T-cell clones might govern longterm tolerance. "At the time of these studies there wasn't a method to identify alloreactive $\mathrm{T}$ cells, as the alloresponse was considered to involve thousands of different T-cell specificities," says Sykes. "As such, there was no way to distinguish between clonal deletion and anergy or specific unresponsiveness of donor-reactive T cells. We therefore sought to develop an assay to distinguish between these mechanisms."

In their recent study, the researchers identified recipient CD4 and CD8 T cells and sequenced their $\mathrm{T}$-cell receptor (TCR) $\beta$-chain CDR3 regions. "A pretransplant mixed lymphocyte reaction-in which recipient cells were labelled with a fluorescent dye and incubated with irradiated donor cells that had been labelled with another dyefollowed by sorting of the recipient cells that had divided, allowed us to identify the donor-reactive TCRs by comparing the sequences of the $\mathrm{T}$ cells that had proliferated with those of the unstimulated pretransplant cell populations," explains Sykes. "The clones that had expanded, compared with the unstimulated population, provided a 'fingerprint' of the donor-reactive T-cell repertoire. We then counted and compared these clones in unstimulated post-transplant T-cell populations."

Three tolerant patients with CKBMTs had a reduced number of circulating T-cell clones that were identified pretransplantation as donorreactive. Such a reduction was not observed in a CKBMT recipient who received the same tolerance protocol but failed to achieve tolerance. Two recipients of kidney transplants alone exhibited post-transplantation expansion of circulating T-cell clones that were identified as donor-reactive before transplantation. Although performed in a small group of patients, the researchers suggest that their findings implicate clonal deletion as a mechanism of tolerance. "Our assay might have considerable potential as a specific biomarker that could noninvasively predict and diagnose rejection," proposes Sykes. "Moreover, it might help identify patients who are undergoing spontaneous allograft tolerance, and ultimately be used to detect patients who are likely to be successfully weaned from immunosuppression. These hypotheses all require further analyses."

Jessica K. Edwards

Original article Morris, H. et al. Tracking donorreactive T cells: Evidence for clonal deletion in tolerant kidney transplant patients. Sci. Transl. Med. doi: 10.1126/scitransImed.3010760

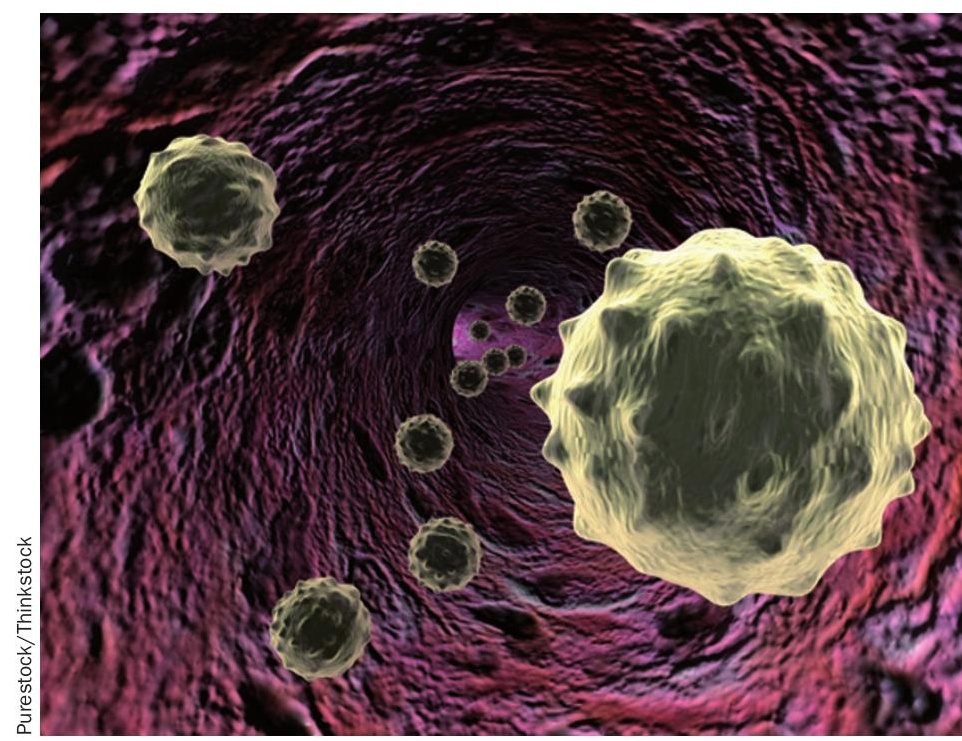

